\title{
Quality of Life in Fibromyalgia Patients with Craniomandibular Disorders
}

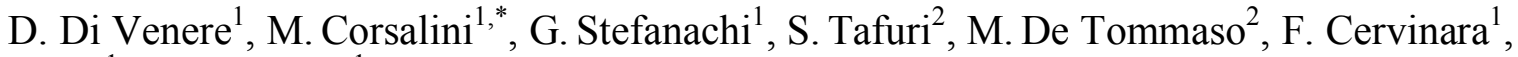 \\ A. $\operatorname{Re}^{1}$ and F. Pettini ${ }^{1}$ \\ ${ }^{I}$ Dental School, University of Bari, Bari, Italy; ${ }^{2}$ Department of Biomedical Sciences and Human Oncology, University of \\ Bari, Bari, Italy
}

\begin{abstract}
Fibromyalgia (FM) is a rheumatic disease which affects fibrous tissues and muscles; it is characterized by chronic pain and it is often associated with craniomandibular disorders (CMD). 31 patients were assessed from March 2012 to October 2012 through the administration of specific questionnaires and following neurologic and gnatologic assessment. A relevant correlation between FM and CMD emerges from the present study, as $80.6 \%$ of our patients report CMD symptoms with high prevalence of myofascial pain (84\%). Multivariate regression analysis revealed that the patients in the present study did not differ in score of quality of life questionnaires from patients with fibromyalgia. The neuropathic pain diagnostic questionnaire (DN4) scores were positively affected by belonging to group II of Research Diagnostic Criteria of Temporomandibular Disorders (RDC/ TDM) classification, suggesting the possibility of a neuropathic component in chronic pain in this CMD group, as already speculated in our study on the correlation between burning mouth syndrome and CMD and by other authors in studies on chronic low back pain. However, further clinic and instrumental studies are needed in order to test this assumption.
\end{abstract}

Keywords: Craniomandibular disorders, fibromyalgia, multivariate regression, myofascial pain, quality of life, temporomandibular disorders.

\section{INTRODUCTION}

Fibromyalgia (FM), according to the American College of Rheumatology (ACR) [1] "is a chronic musculoskeletal pain syndrome of unknown etiology, characterized by widespread pain for more than 3 months and tenderness in at least 11 tender point sites out of 18 ".

Other symptoms are physical and mental tiredness, insomnia and neuropsychological problems [1].

FM is a common disease with a prevalence of about $2 \%$, that affects women more than men $(3.4 \%$ versus $0.5 \%)$ $[2,3]$.

The causes of FM are unknown, although it is generally agreed that the persistence of musculoskeletal pain is due to the so called 'mechanism of central sensitization' [4-6]. De Tommaso [7] defined central sensitization as "a phenomenon present in any type of pain, nociceptive or neuropathic, where a noxious stimulus, able to recruit the $\mathrm{C}$ and A-delta sensory fibers, reduces in a short times the pain threshold in the primarily involved areas, a phenomenon known as hyperalgesia, while in the adjacent zones any mechanical input becomes painful, as an effect of the so-called "allodynia".

Craniomandibular disorders (CMD) display a wide range of medical cases concerning masticatory muscles, temporomandibular joints or both. The patient affected by craniomandibular disorders can suffer pain in every component of the oro-facial district (pre-auricular zone, masticatory

*Address correspondence to this author at the Dental School, University of Bari, Bari, Italy; Tel: (+39) 338-2062803;

E-mail: massimo.corsalini@uniba.it muscles, temporomandibular joint) and presents a limited mouth opening with or without latero-deviation and joint noise [8].

CMD are widespread in the population: prevalence studies have reported that approximately $33 \%$ of the population have CMD signs and 75\% have CMD symptoms [8, 9].

Dworkin e LeResche [10] Research Diagnostic Criteria of Temporomandibular Disorders (RDC/ TDM) classified craniomandibular disorders into three subgroups:

Group 1:

- 1a: myofascial pain;

- 1b: myofascial pain with limited opening;

Group 2:

- 2a: disk displacement with reduction;

- 2b: disk displacement without reduction, with limited opening;

- 2c: disk displacement without reduction without limited opening;

Group 3:

- 3a: arthralgia;

- 3b: osteoarthritis;

- 3c: osteoarthrosis.

CMD and FM were once considered unrelated entities, but several studies [11-15] highlighted oro-facial symptoms in patients affected by FM indicating that there could be a correlation between these two conditions [16]. 
Myofascial face pain, for example, was considered to be a regional expression of fibromyalgia [17]. Yunus [18] classified fibromyalgia, irritable bowel syndrome, myogenic temporomandibular disorders, tension-type headache, migraine and other chronic syndromes under the name "Central Sensitivity Syndromes (CSS)" characterized by the same underlying pathophysiology, i.e. a prolonged central sensitization [6].

The aim of our work was to determine the prevalence of CMD among patients with FM and CMD, classifying craniomandibular symptoms within the Dworkin and LeResche's subgroups. FM syndrome and CMD are both chronic diseases and different authors studied the effects of chronic pain on the quality of life among patients affected by this $[19,20]$.

Gijsen et al. [21] claimed that "longitudinal studies suggest that the cumulative effect of comorbid conditions is not simply additive: certain combinations of diseases may have a greater effect on quality of life than others". Therefore the second aim of our study was to determine the burden of CMD symptoms on quality of life among FM patients, testing the hypothesis according to which fibromyalgia subjects affected by craniomandibular disorders have poorer quality of life than fibromyalgia patient without CMD.

\section{MATERIALS AND METHODS}

This study was completed between March and October 2012 at the Neurophysiopathology of Pain Unit, Bari University Hospital. All the participants filled in an informed consent form; the Ethics Committee of the Policlinico General Hospital approved this study. Of the 100 patients examined in the above mentioned facility, reporting chronic pain not ascribable to any pathology, 55 received a fibromyalgia diagnosis. Of these 55 patients, 31 were willing to undergo a gnatologic visit and to fill in questionnaires aiming at the assessment of their quality of life (Fig. 1).

100 subjects were visited at the Neurophysiopathology of Pain Unit, Bari University Hospital.

\begin{tabular}{|l|l|}
\hline INCLUSION CRITERIA & EXCLUSION CRITERIA \\
\hline \hline $\begin{array}{l}\text { - widespread pain for at least } 3 \text { months; } \\
\text { a minimum of } 11 \text { out of } 18 \text { tender points painful in } \\
\text { response to digital palpation in } 9 \text { matched areas in } \\
\text { the body }\end{array}$ & $\begin{array}{l}\text { patients in whom a diagnosis of widespread pain } \\
\text { could be ascribed to a different disease from } \\
\text { fibromyalgia; } \\
\text { patients receiving pharmacological treatment }\end{array}$ \\
\hline
\end{tabular}

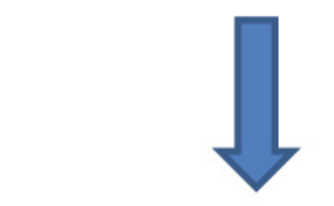

55 fibromyalgic patients were selected

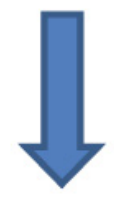

31 fibromyalgic patients accepted to undergo the complete examination:

\section{Neurological assessment}

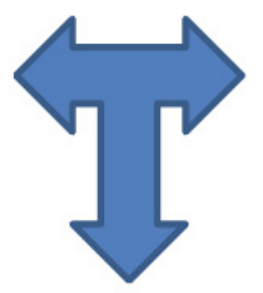

Quality of lifeexamination

Fig. (1). Diagram of Materials and methods section. 


\section{Clinical-gnatologic Assessment}

For each sample component age, gender and profession. pain severity and frequency information were measured and reported. A neurological examination was carried out followed by a clinical-gnatologic assessment verifying the presence of widespread pain also on the orofacial region level and the presence of CMD signs and symptoms. In the first examination, the quality of life of fibromyalgia patients was measured using various instruments.

\section{Neurological Examination}

Patients were submitted to a standardized manual tender point survey [22]: "tender point count was defined as the number of positive tender points" (tender pn), while "total tender point score was calculated by adding individual scores for all 18 tender points"(tender $p$ tot).

At the end patients answered a neuropathic pain diagnostic questionnaire (DN4), a validated tool to identify neuropathic pain on a score equalling $\geq 4$ out of 10 [23].

\section{Clinical-gnatologic Assessment}

All fibromyalgia patients underwent a visit of the orofacial district according to the RDC/TMD guidelines [10].

Orofacial pain was measured with a ten centimetres visual analogue scale (VAS).

Presence of oral parafunctions like bruxism, clenching, lip and cheek biting etc was also observed.

\section{Quality of Life Assessment}

Quality of life was measured by means of the following instruments: Medical Outcome Short Form Health Survey (SF-36) and the Fibromyalgia Impact Questionnaire (FIQ).

SF-36 is a self-administered questionnaire composed of 36 questions regarding eight health concepts: physical functioning, physical role limitations, emotional role limitations, bodily pain, general health perceptions, vitality, social functioning and general mental health [24].

The answers to questionnaires lead, summing all values, to two measures: SF-36 ISF which includes the values regarding difficulties in physical activity and SF-36 ISM which includes the values about psychological and mental components. Higher scores represent a better functioning [19].

FIQ, according to Burckardt et al. [25], "is a selfreported instrument that measures the current health status of the FM patient"; it includes 13 items assessing the difficulty in performing common, everyday life activities, general health, working activity and 7 items assessing the intensity/severity of symptom on a VAS scale from 0 to 10 .

The final score may vary from a minimum of 0 to a maximum of 100 (higher scores represent a higher impact of the disease on the patient's quality of life) [25].

In the present study we adopted a 0-70 simplified score system that includes only 7 items assessing the intensity/severity of symptom (with 70 indicating greater impairment).

\section{Statistical Analysis}

For each of the selected patients a file was filled in, it included demographic variables, pain duration at t0, psychological tests (SF-36 ISF, SF-36 ISM, FIQ), VAS score, DN 4 Questionnaire outcomes, number of tender points, pain frequency and location, parafunctions, CMD group to which the patient was assigned in accordance with the RDC/TDM classification. The files were then put in a database created with File Marker Pro software and analyzed with STATA MP11 software. The quantitative variables were expressed as means \pm standard deviation; qualitative variables as proportions. As to assess the effect of age, gender, pain duration at t0, VAS, tender point, pain frequency and location, presence of parafunctions, CMD group on the outcomes of psychometric tests about the quality of life, multivariate regression models were set. For all the used tests a value of $\mathrm{P}<0.05$ was considered substantial.

\section{RESULTS}

We studied 31 patients, 3 males $(9.7 \%)$ and 28 females $(90.3 \%)$, their age ranged from 20 to 65 years (average age $47.9 \pm 9.9$ years).

The average pain duration, at the time patients were hired, was $8.1 \pm 8.0$ months. 18 patients $(58.1 \%)$ reported a daily pain frequency, $11(35.5 \%)$ a weekly frequency and 2 $(6.4 \%)$ a monthly frequency.

In 25 FM patients (80.6\%) symptoms and signs of craniomandibular disorders were observed.

18 patients $(58.1 \%)$ had parafunctions, more in detail, 12 (38.7\%) were affected by bruxism, 6 of whom (19.3\%) used the bite.

In Fig. (2) we reported the distribution of craniomandibular disorders diagnosis according the RDC/TMD classification Summarizing, a muscular disorder is present in 21 patients $(84 \%)$, a disc dislocation in 11 patients $(44 \%)$, inflammatory and degenerative joint disorders in 3 patients (12\%). In $40 \%$ of cases we have a combined diagnosis.

Descriptive statistics were performed to summarize sample characteristics in Table $\mathbf{1}$.

In Table $\mathbf{2}$ are presented the results of a comparison of quality of life assessment between FM patients affected by craniomandibular disorders and FM subjects without any symptoms.

As we note, quality of life scores for all domains of the SF-36 were similar between patients groups.

Multivariate regression estimates that FIQ scale scores are not affected by the presence of temporomandibular disorders but by the tender point total score (coefficient 0.21 ; $\mathrm{t}=2.5 ; \mathrm{p}=0.03)$. DN4 questionnaires scores are negatively affected by the presence of parafunctions (coefficient $=-3.7$; $\mathrm{t}=2.38 ; \mathrm{p}=0,038$ ) and by the belonging to group $\mathrm{I}$ of RDC/TDM classification; whereas the above mentioned scores are positively affected by the belonging to group II ( $\operatorname{coef} 3.2 ; \mathrm{t}=3.27 ; \mathrm{p}=0.008$ ).

\section{DISCUSSION}

Our results suggest that craniomandibular disorders are pervasive among the FM population, as $80.6 \%$ of our 


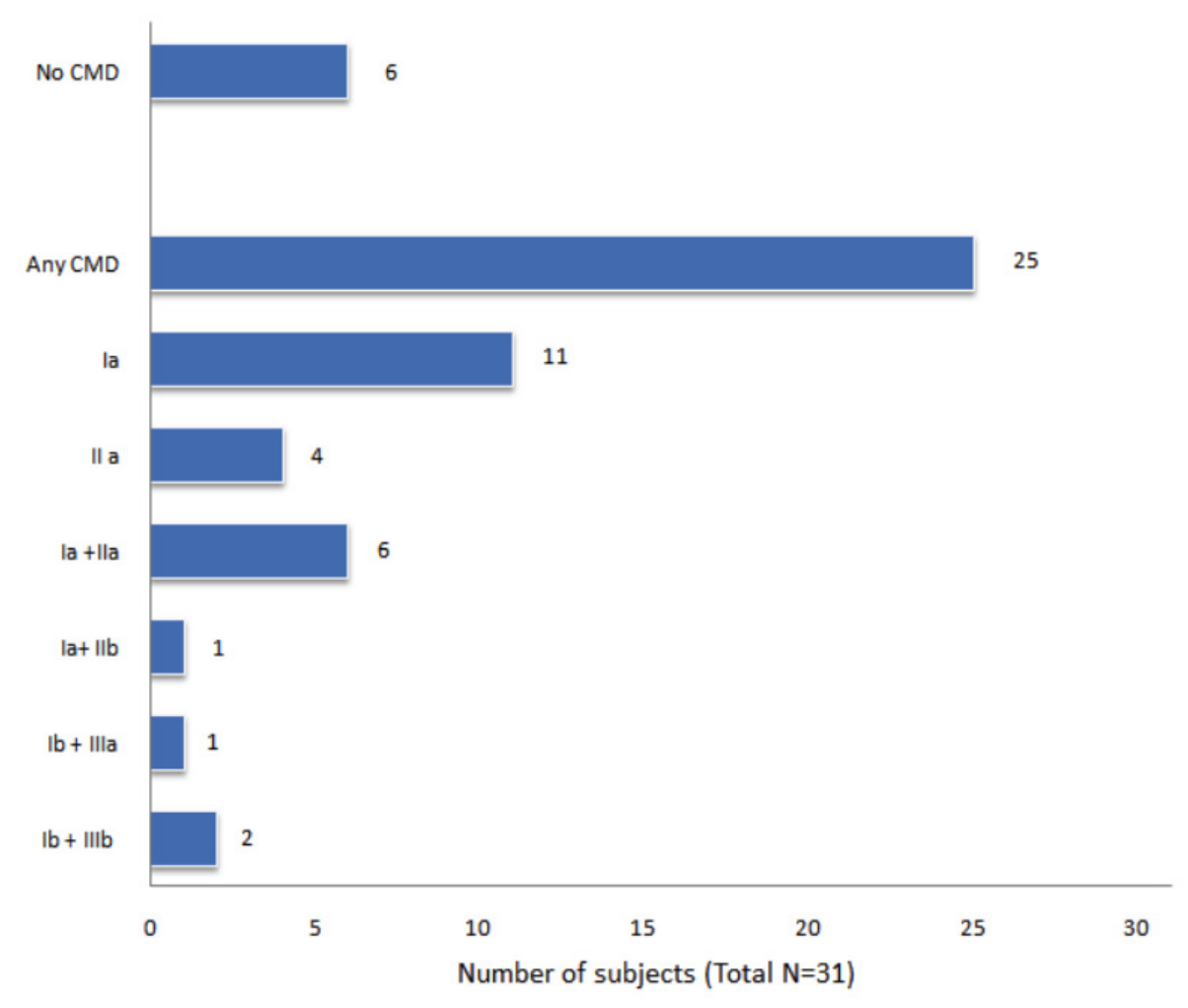

\section{Distribution of CMD diagnoses}

Fig. (2). Distribution of CMD diagnoses.

patients report CMD symptoms with high prevalence of myofascial pain $(84 \%)$, alone or associated to disc dislocation or to arthralgia. These results are consistent with Plesh et al. [11] who reported that $75 \%$ of subjects with FM suffered from muscle-related CMD.

Table 1. Sample characteristics.

\begin{tabular}{|c|c|c|}
\hline Evaluation & Mean & Standard deviation \\
\hline \hline DN4 & 4.9 & 2.6 \\
\hline VAS & 7.9 & 1.8 \\
\hline Tender pn & 15.3 & 4.3 \\
\hline Tender p tot & 94.0 & 43.4 \\
\hline SF-36 ISF & 29.8 & 8.5 \\
\hline SF-36 ISM & 37.4 & 11.8 \\
\hline FIQ & 54.2 & 13.9 \\
\hline
\end{tabular}

Myofascial pain in the masticatory muscles is a significant component in fibromyalgia according several authors.

Hedenberg-Magnusson et al. [26] in their study found that $94 \%$ of 191 patients with FM also displayed symptoms of CMD. Fricton [14] in 2004 speculated that "clinical overlap between these conditions may reflect a shared underlying pathophysiologic basis" [14, 15]. Woolf [6] and Yunus [18] defined FM and myogenic temporomandibular disorders as "overlapping conditions" that are, together other diseases, "collectively known as central sensitivity syndromes".

Table 2. Results of comparison between FM patients with CMD and FM subjects without any symptoms Differences between groups are significant at $\mathbf{p}<0.05$. N.S.: Not significative.

\begin{tabular}{|c|c|c|}
\hline $\begin{array}{c}\text { Characteristic, } \\
\text { means (SD) }\end{array}$ & $\begin{array}{c}\text { FM patients with } \\
\text { CMD (N=25) }\end{array}$ & $\begin{array}{c}\text { FM patients without } \\
\text { CMD (N=6) }\end{array}$ \\
\hline \hline SF-36 ISF & $29.8 \pm 8.9$ N.S. & $29.5 \pm 6.9$ \\
\hline SF-36 IFM & $36 \pm 10.5$ N.S. & $43.1 \pm 16.1$ \\
\hline FIQ & $54 \pm 14.3$ N.S. & $53.1 \pm 12.2$ \\
\hline
\end{tabular}

According to these authors, there is a strong evidence of central sensitization in myofascial pain, "including decreased pain threshold by various nociceptive stimuli at sites remote from painful areas, accentuated nociceptive spinal flexion reflex and augmented cortical activation by functional magnetic resonance imaging" [18].

An important consequence of this is relevant to the treatment of these pathologies: "since total disease burden with functional impairment is greater in patients with many associated conditions, a practicing physician should treat these conditions for optimal results" [27, 28]. 
The second aim of our study was to determine the burden of CMD symptoms on quality of life among patients with FM.

Quality of life has been described by the World Health Organization as "an individual's perception of their position in life in the context of the culture and value system of which they live with the relation to their goals, expectations, standards and concerns" [29].

Several studies suggest that certain combinations of comorbid conditions may have a greater effect on quality of life than others [21]. In our study, the quality of life scores were similar in fibromyalgia patients with or without CMD: FM totally contributed to the physical and mental disability in affected individuals that we examined. Also Marcus et al. [30] in their epidemiological study on fibromyalgia and headache found that migraine did not affect the perception fibromyalgic patients had of their disease. Hoffman and Dukes [31], in an article on the health status of patients with fibromyalgia examined the extent to which FM contributed to the overall health status burden of patients with and without a specific concurrent condition and they found that "while health status in general population deteriorated in conjunction with the number of pain condition present, FM had a remarkably consistent pattern of health status impairment even that it occurred alone".

In other words, patients with FM are already so burdened by their condition that the introduction of an additional pain condition (as masticatory muscle pain in our patients) does not affect their health related quality of life (HRQoL) [32].

Multivariate regression analysis revealed that the DN4 questionnaires score are positively affected by the belonging to group II of RDC/TMD classification. DN4 questionnaire is a validated tool to identify neuropathic pain and this suggests the possibility of a neuropathic component in chronic pain in this CMD group (disk displacement with and without limited opening) as already speculated in chronic low back pain $[33,34]$ and in our study on the correlation between BMS and TMD [35].

Limitations of this study are the small sample size and that all health status data were based on self-report. Further clinic and instrumental analysis (such as a standardized protocol of quantitative sensory testing and laser-evoked potentials) are needed in order to test the hypothesis of a neuropathic component of pain due to craniomandibular disorders.

\section{CONCLUSION}

The results of the present study suggest the existence of a close association between FM and CMD. A high percentage of FM patients $(80.6 \%)$ indeed resulted to be positive to at least one RDC/TMD diagnosis.

We therefore suggest to subject FM patients to a gnatologic assessment in order to complete their diagnostic situation.

On the other hand the dentist should be able to perform a differential diagnosis between localized or systemic muscle disorders, as to better handle patients with orofacial pain. In fact, once identified, TMD patients who have co-morbid conditions such as fibromyalgia, can best be treated by an interdisciplinary team of clinicians.

\section{CONFLICT OF INTEREST}

The authors confirm that this article content has no conflict of interest.

\section{ACKNOWLEDGEMENTS}

Declared none.

\section{REFERENCES}

[1] Wolfe F, Smythe HA, Yunus M, et al. The American College of Rheumatology 1990 Criteria for the Classification of Fibromyalgia. Report of the Multicenter Criteria Committe. Arthitis Rheum 1990; 33:160-72.

[2] Lawrence RC, Felson DT, Helmick CG, et al. Estimates of the prevalence of arthritis and other rheumatic conditions in the United States. Part II. Arthritis Rheum 2008; 58(1): 26-35.

[3] Croft P. Testing for tenderness: What's the point? J Rheumatol 2000; 27: 2531-3.

[4] Staud R, Rodriguez ME. Mechanisms of disease: pain in fibromyalgia syndrome. Nat Clin Pract Rheumatol 2006; 2: 90-8.

[5] Treede RD, Meyer RA, Raja SN, Campbell JN. Peripheral and central mechanism of cutaneous hyperalgesia. Prog Neurobiol 1992; 38: 397-421.

[6] Woolf CJ. Central sensitization:implications for the diagnosis and treatment of pain. Pain 2011; 152: S2-S15.

[7] De Tommaso M, Federici A, Serpino C, et al. Clinical features of headache patients with fibromyalgia comorbidity. J Headache Pain 2011; 12: 629-38.

[8] Carlsson GE. Epidemiology and treatment need for temporomandibular disorders. J Orofac Pain 1999; 13: 232-7.

[9] Rugh JD, Solberg WK. Oral health status in the United States: Temporomandibular disorders. J Dent Educ 1985; 49: 398-406.

[10] Dworkin SF, LeResche L. Research diagnostic criteria for temporomandibular disorders: review, criteria, examinations and specifications, critique. J Craniomand Disord 1992; 6: 301-55.

[11] Plesh O, Wolfe F, Lane N. The relationship between fibromyalgia and temporomandibular disorders: prevalence and symptom severity. J Rheumatol 1996; 11: 1948-52.

[12] Leblebici B, Pektas ZO, Ortancil O, Hurcan EC, Bagis S, Akman $\mathrm{MN}$. Coexistence of fibromyalgia, temporomandibular disorder, and masticatory myofascial pain syndromes. Rheumatol Int 2007; 27: 541-4.

[13] Salvetti G, Manfredini D, Bazzichi L, Bosco M. Clinical features of the stomatognathic involvement in fibromyalgia syndrome: A comparison with temporomandibular disorders patients. Cranio 2007; 25:127-33

[14] Fricton JR. The relationship of temporomandibular disorders and fibromyalgia: implications for diagnosis and treatment. Curr Pain Headache Rep 2004; 8: 355-63.

[15] Moldofsky HK. Disordered sleep in fibromyalgia and related myofascial facial pain conditions. Dent Clin North Am 2001; 45: 70113.

[16] Stohler CS. Muscle-related temporomandibular disorders. J Orofac Pain 1999; 13: 273-84.

[17] Marbach JJ. Is myofascial face pain a regional expression of fibromyalgia? J Muscoskel Pain 1995; 3(2): 93-7.

[18] Yunus MB. The prevalence of fibromyalgia in other chronic pain conditions. Pain Res Treat 2012; 2012: 584573.

[19] Kempen GI, Ormel J, Brilman EI, Relyved J. Adaptive responses among. Dutch elderly: the impact of eight chronic medical conditions on health-related quality of life. Am J Public Health 1997; 87: 34-44.

[20] Schlenk EA, Erlen JA, Dunbar-Jacob J, et al. Health-related quality of life in chronic disorders: a comparison across studies using the MOS SF-36. Qual Life Res 1998; 7(1): 57-65

[21] Gijsen R, Hoeymans N, Schellevis FG, et al. Causes and consequences of comorbidity: A review. J Clin Epidemiol 2001; 54(7): 661-74. 
[22] Okifuji A, Turk DC, Sinclair JD, Startz TW, Marcus DA. A standardized manual tender point survey. I. development and determination of a threshold point for the identification of positive tender points in fibromyalgia syndrome. J Rheumatol 1997; 24: 377-83.

[23] Bouhassira D, Attal N, Alchaar H, et al. Comparison of pain syndromes associated with nervous or somatic lesions and development of a new neuropathic pain diagnostic questionnaire (DN4). Pain 2005; 114: 29-36.

[24] Ware JE, Sherbourne CD. The MOS 36-item short-form health survey (SF-36) 1: conceptual framework and item selection. Med Care 1992; 30(6): 473-83.

[25] Burckardt CS, Clark SR, Bennett RM. The Fibromyalgia Impact Questionnaire: development and validation. J Rheumatol 1991; 18: 728-33.

[26] Hedenberg-Magnusson B, Ernberg M, Kopp S. Symptoms and signs of temporomandibular disorders in patients with fibromyalgia and local myalgia of the temporomandibular system. A comparative study. Acta Odontol Scand 1997; 55(6): 344-9.

[27] Balasubramaniam R, de Leeuw R, Zhu Hua, Nickerson R, Okeson $\mathrm{P}$, Carlson C. Prevalence of temporomandibular disorders in fibromyalgia and failed back syndrome patients: a blinded prospective comparison study. Oral Surg Oral Med Pathol Oral Radiol Endod 2007; 104: 206-16.

[28] Velly AM, Look JO, Schiffman E, et al. The effect of fibromyalgia and widespread pain on the clinically significant temporomandibu- lar muscle and joint pain disorders: a prospective 18-month cohort study. J Pain 2010;11:1155-64.

[29] The World Health Organization quality of life assessment (WHOQOL): Position paper from the World Health Organization. Soc Sci Med 1995; 10: 1403-09.

[30] Marcus DA, Bernstein C, Rudy TE. Fibromyalgia and headache: an epidemiological study supporting migraine as part of the fibromyalgia syndrome. Clin Rheumatol 2005; 24: 595-601.

[31] Hoffman DL, Dukes EM. The health status burden of people with fibromyalgia: a review of studies that assessed health status with the SF-36 or the SF-12. Int J Clin Pract, 2008; 62: 115-26

[32] Wagner JS, DiBonaventura M, Chandran AB, Cappelleri C. The association of sleep difficulties with health-related quality of life among patients with fibromyalgia. BMC Musculoskelet Disord 2012; 13: 199-207.

[33] Attal N, Perrot S, Fermanian J, Bouhassira D. The neuropathic components of chronic low back pain: a prospective multicenter study using the DN4 Questionnaire. J Pain 2011; 12(10): 1080-7.

[34] Brisby H. Pathology and possible mechanism of nervous system response to disc degeneration. J Bone Joint Surg Am 2006; 88(supp12): 68-71.

[35] Corsalini M, Di Venere D, Pettini F, Lauritano D, Petruzzi M. Temporomandibular Disorders in Burning Mouth Syndrome Patients: An Observational study. Int J Med Sci 2013; 10(12): 1784-9.

Received: November 08, 2014

Revised: December 11, 2014

Accepted: December 15, 2014

(C) Di Venere et al.; Licensee Bentham Open.

This is an open access article licensed under the terms of the Creative Commons Attribution Non-Commercial License (http://creativecommons.org/licenses/by-nc/3.0/) which permits unrestricted, non-commercial use, distribution and reproduction in any medium, provided the work is properly cited. 